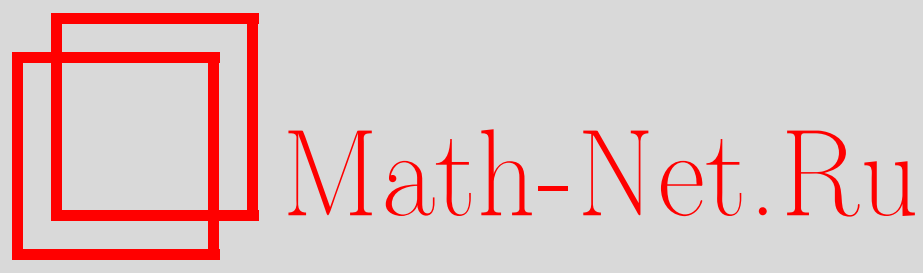

Ю. Н. Скрябин, Теория рассеяния нейтронов в магнетиках, ТМФ, 2011, том 168, номер 3, 551-570

DOI: https://doi.org/10.4213/tmf6700

Использование Общероссийского математического портала Math-Net.Ru подразумевает, что вы прочитали и согласны с пользовательским соглашением http://www . mathnet.ru/rus/agreement

Параметры загрузки :

IP: 3.85 .5 .30

26 апреля 2023 г., 15:54:21

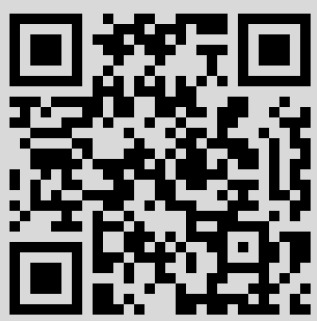




\title{
ТЕОРИЯ РАССЕЯНИЯ НЕЙТРОНОВ В МАГНЕТИКАХ
}

\begin{abstract}
Дается обзор теоретических исследований магнитоупорядоченных веществ с помощью рассеяния медленных нейтронов. Рассмотрены методы расшифровки магнитных структур с использованием теории симметрии (неприводимых представлений пространственных групп) и теория малоуглового рассеяния нейтронов на крупномасштабных неоднородностях в магнитных материалах.
\end{abstract}

Ключевые слова: волновой вектор магнитной структуры, пространственные группы, неприводимые представления, базисные функции, дифракция нейтронов, малоугловое рассеяние, нейтронная оптика.

\section{1. ВВЕДЕНИЕ}

Исследование статистической механики ферромагнетизма началось с классической работы Блоха, в которой было предположено, что энергетический спектр ферромагнетика вблизи основного состояния может быть аппроксимирован спектром идеального газа бозевских квазичастиц - спиновых волн. С другой стороны, хорошо известно, что при высоких температурах работает приближение молекулярного поля Вейсса. Тогда же стало ясно, что невозможно построение универсальной теории, пригодной для описания ферромагнитного состояния во всем интервале температур существования спонтанного момента вплоть до температуры Кюри. В результате сложились две независимые области теоретического исследования ферромагнитного состояния: область низких температур и высоких (окрестность температуры фазового перехода), между которыми долгое время существовал ничем не заполненный разрыв.

Первая удачная попытка была предпринята Тябликовым [1] на основе расцепления двухвременнь́х функций Грина [2], [3]. Тябликов показал, что можно ввести приближенное описание ферромагнетика в широком интервале температур (включая и промежуточную температурную область) в терминах “спиновых волн", энергия которых, однако, должна зависеть от температуры. Этот результат затем был подтвержден экспериментально на многих ферромагнетиках с помощью метода неупругого рассеяния нейтронов [4].

* Институт физики металлов УрО РАН, Екатеринбург, Россия. E-mail: skryabin@imp.uran.ru 
Следует отметить, что при получении фундаментальных теоретических результатов относительно ферромагнитного состояния использовались каждый раз различные математические подходы, специфичные для поставленной узкой задачи. В известной монографии Тябликова [5], [6] дан обзор методов, широко используемых в те годы в теории ферромагнетизма. Между тем в середине прошлого века наметились пути создания в статистической механике ферромагнетизма единого метода, основанного на диаграммной технике для спиновых операторов.

Важный шаг в этом направлении был сделан в работах [7], [8], где на основе представления спиновых операторов квадратичной формой ферми- (или бозе-) операторов проблема гейзенберговского ферромагнетика была сведена к изучению системы взаимодействующих ферми- или бозе-частиц [9], усложненной необходимостью специального учета фиктивных состояний, вводимых этим представлением. В результате были установлены границы применимости результата Тябликова, полученного методом расцепления функций Грина, вычислено затухание зависящих от температуры спиновых волн в окрестности точки Кюри. Кроме того, было дано доказательство “теоремы Вика" для спиновых операторов. Это позволило построить диаграммную технику для гейзенберговского ферромагнетика непосредственно в спиновых операторах без предварительного сведения их к ферми- или бозе-операторам. Подробное описание диаграммной техники для спиновых операторов изложено в книгах [10], [11], а диаграммной техники для $X$-операторов модели Хаббарда - в книге [12].

Однако реальный мир магнитных структур оказался гораздо разнообразнее простого ферромагнетизма с параллельным упорядочением атомных магнитных моментов, и открыть этот мир позволил метод дифракции медленных нейтронов, который тесно связан с методом функций Грина.

В 1966 г. на основе построенного в Белоярке исследовательского реактора ИВВ-2 начал свою работу Уральский нейтронный центр, где за несколько десятилетий был выполнен ряд первоклассных работ по теории рассеяния медленных нейтронов в магнитоупорядоченных кристаллах, созданы новые методы расшифровки магнитных структур с использованием теории симметрии, экспериментально определены магнитные структуры ряда интересных магнетиков, созданы методики исследования магнитного состояния сплавов переходных металлов с помощью малоуглового рассеяния, разработаны новые методики исследования магнитного состояния вещества с помощью поляризованных нейтронов.

Цель настоящей статьи - дать обзор результатов ряда теоретических исследований по нейтронографии, проводимых в Уральском нейтронографическом центре. Теоретические исследования цитируются в основном по известным книгам [4], [13]-[15] и обзорам [16]-[18], в которых можно найти все ссылки на оригинальные работы.

\section{2. ИСПОЛЬЗОВАНИЕ СИММЕТРИИ ПРИ НЕЙТРОНОГРАФИЧЕСКОМ ИССЛЕДОВАНИИ МАГНИТНЫХ СТРУКТУР КРИСТАЛЛОВ}

2.1. Определение волновых векторов структуры. Первые плодотворные попытки систематизации магнитных структур кристаллов связаны с изучением их собственной симметрии как системы пространственно-упорядоченных аксиальных 
векторов (атомных магнитных моментов). Такой подход представляет собой естественное развитие теории симметрии кристаллических структур как пространственно-упорядоченных систем точек (атомов). Известно, что все разнообразие кристаллических структур может быть описано с помощью 230 федоровских (пространственных) групп. Полностью описать какую-либо кристаллическую структуру значит задать ее пространственную группу и позиции кратных точек, занимаемых в ней атомами, т. е., по существу, положение атомов в элементарной ячейке.

Пространственная группа кристалла $G$ представляет собой групповую совокупность операций симметрии, состоящих из трансляций, поворотов, инверсии и т.д., оставляющих этот кристалл инвариантным. Для описания магнитных структур было предложено добавить к перечисленным выше чисто кристаллографическим элементам симметрии одну дополнительную операцию $R$ - обращение спина, т. е. изменение направления спина на противоположное. Построенные с помощью элементов пространственных групп и операции $R$ групповые совокупности называются шубниковскими группами. Число шубниковских групп, призванных описывать симметрию магнитоупорядоченных кристаллов, равно 1651 (включая и 230 федоровских групп, описывающих симметрию парамагнитных кристаллов). Указать магнитную структуру кристалла - значит задать его шубниковскую группу и позиции кратных точек в ней, т. е., по существу, задать ориентацию атомных спинов в элементарной ячейке.

Такой подход до определенного времени описывал все известные магнитные структуры и казался вполне удовлетворительным. До открытия геликоидальных и так называемых несоизмеримых магнитных структур все магнитные структуры могли быть охарактеризованы элементарной магнитной ячейкой. Нейтронографические исследования 50-х гг. ХХ в. привели к открытию множества магнитных структур с увеличением магнитной ячейки в два, четыре, восемь раз по сравнению с химической ячейкой кристалла. Магнитные структуры такого типа прекрасно описываются аппаратом шубниковской симметрии. Важным при этом оказывается понятие магнитной решетки.

Хорошо известно, что кристаллические структуры характеризуются решетками Браве, построенными на основных векторах трансляций $\mathbf{t}_{1}, \mathbf{t}_{2}, \mathbf{t}_{3}$. Каждая из 230 федоровских групп описывается одной из 14 решеток Браве. Магнитная решетка, построенная на основных векторах трансляций $\mathbf{t}_{1}^{\mathrm{m}}, \mathbf{t}_{2}^{\mathrm{m}}, \mathbf{t}_{3}^{\mathrm{m}}$ магнитоупорядоченного кристалла, принадлежит к одной из 36 магнитных (черно-белых) решеток Браве. Понятие магнитной решетки включает в себя понятие антитрансляции, т. е. операции $\mathbf{t} R$, состоящей из обычной трансляции и обращения спина.

Любая решетка шубниковской симметрии описывает коллинеарную магнитную структуру, однако не следует думать, что шубниковские группы не могут описывать неколлинеарные магнитные структуры. Подобно тому как любая сколь угодно сложная кристаллическая структура может рассматриваться как совокупность некоторого числа одинаковых решеток Браве, вложенных друг в друга, многие неколлинеарные магнитные структуры могут рассматриваться как вложенные друг в друга магнитные решетки Браве. Каждая из этих решеток образована коллинеарными спинами, однако относительная ориентация спинов, принадлежащих различным решеткам, может быть произвольной и приводить к неколлинеарной антиферромагнитной структуре. 
Шубниковская симметрия недостаточна при описании магнитных структур, для которых не существует магнитной ячейки; примером таковых являются простая спираль и продольная спиновая волна. В первой имеются ферромагнитные слои, в которых ориентация спинов меняется на некоторый угол при переходе от одного слоя к другому вдоль направления гексагональной оси (в редкоземельных металлах $\mathrm{Dy}, \mathrm{Tb}, \mathrm{Ho})$. Такого типа структуры, получившие названия спиральных, геликоидальных или винтовых, обнаружены во многих десятках различных кристаллов. Вторая структура является примером также часто встречающихся магнитных структур типа спиновой волны. Эта структура коллинеарная, но с изменением проекций атомного спина по гармоническому закону вдоль некоторого направления. Если сохраняющаяся проекция спина ориентирована вдоль направления модуляции, структура называется продольной спиновой волной, а если перпендикулярно к этому направлению - поперечной спиновой волной.

Такого типа магнитные структуры (их часто называют модулированными) не являются редкими, а наблюдаются в различных вариациях во множестве кристаллов различного типа. Поскольку изменение фазы между двумя соседними спинами может быть произвольным, говорят о несоизмеримости периода такой структуры по отношению к кристаллическому, оттуда следует, что их нельзя охарактеризовать конечной элементарной магнитной ячейкой. Для описания их симметрии, а также для ряда других структур, не описываемых аппаратом шубниковских групп, развивались различные обобщения этих групп, получившие общее название цветной симметрии, однако с физической точки зрения этот способ описания магнитных структур мало конструктивен, и успех в описании самого общего типа магнитных структур связан с использованием теории представлений пространственных групп кристаллов. Прежде чем переходить к изложению основной идеи этого метода, рассмотрим вопрос о волновых векторах магнитной структуры.

Информацию о трансляционных свойствах магнитной структуры кристалла удобно выразить с помощью понятия волнового вектора. По определению волновой вектор $\mathbf{k}$ магнитной структуры позволяет связать спин $\mathbf{S}_{n i} i$-го атома в $n$-й примитивной ячейке кристалла со спином $\mathbf{S}_{0 i} i$-го атома в нулевой ячейке посредством соотношения

$$
\mathbf{S}_{n i}=e^{i \mathbf{k t}_{n}} \mathbf{S}_{0 i}
$$

где $\mathbf{t}_{n}-$ вектор трансляции из нулевой ячейки в $n$-ю. Волновой вектор структуры является всегда одним из волновых векторов первой зоны Бриллюэна и может быть представлен в виде

$$
\mathbf{k}=h \mathbf{b}_{1}+k \mathbf{b}_{2}+l \mathbf{b}_{3},
$$

где $\mathbf{b}_{1}, \mathbf{b}_{2}, \mathbf{b}_{3}$ - основные векторы обратной решетки кристалла, a $h, k, l$ - некоторые числа такие, что вектор (2) всегда лежит в первой зоне.

Напомним, что звездой волнового вектора называется совокупность неэквивалентных лучей, получаемых из данного волнового вектора (луча $\left\{\mathbf{k}_{1}\right\}$ ) действием всех элементов симметрии точечной группы кристалла. Если обозначить элементы, порождающие неэквивалентные лучи $\left\{\mathbf{k}_{L}\right\}$, через $h_{L}$, то вся звезда может быть получена с помощью соотношения

$$
\mathbf{k}_{L}=h_{L} \mathbf{k}_{1}
$$


где $L=1,2, \ldots, l_{\mathbf{k}}-$ число лучей звезды.

Ясно, что многолучевые звезды появляются в том случае, когда волновой вектор лежит в направлении, для которого имеются кристаллографические эквивалентные направления. Если волновой вектор принадлежит многолучевой звезде, то возможна суперпозиция состояний, описываемых одним лучом.

В случае многолучевой звезды магнитная структура должна характеризоваться всеми лучами этой звезды, и вместо соотношения (1) мы должны иметь

$$
\mathbf{S}_{n i}=\sum_{L} e^{i \mathbf{k}_{L} \mathbf{t}_{n}} \mathbf{S}_{0 i}^{L}
$$

Векторы $\mathbf{S}_{0 i}^{L}$, которые могут быть комплексными, назовем лучевыми вкладами в магнитную структуру.

Фундаментальное соотношение (4) определяет трансляционные свойства произвольной магнитной структуры, если задана звезда волнового вектора. Однако эти свойства зависят не только от звезды, но также и от того, какие лучевые вклады $\left\{\mathbf{S}_{0 i}^{L}\right\}$ отличны от нуля. Назовем совокупность лучей звезды, для которых лучевые вклады отличны от нуля, каналами перехода. Нетрудно увидеть, что именно канал перехода однозначно задает трансляционные свойства магнитной структуры. Возникает, таким образом, следующая теоретическая задача: указать все магнитные решетки, которые могут получиться из данной кристаллической решетки путем магнитного упорядочения.

Для ее решения следовало бы взять различные по симметрии звезды волнового вектора для каждой из 14 решеток Браве (список всех таких звезд конечен и содержится в справочниках, например в монографии Ковалева [19]) и для всех каналов каждой звезды (комбинируя различным образом ее лучи) найти по соотношению (4) соответствующую им решетку. Для всех лифшицевских звезд [20] (волновые векторы которых оканчиваются в симметричных точках зоны Бриллюэна) такая задача решена [21], [22] и результаты сведены в таблицу, по которой для заданного канала перехода в исходной решетке можно указать магнитную решетку. Оказалось, что во всех каналах лифшицевских звезд возникает одна из 36 магнитных решеток шубниковской симметрии. Таким образом, указание магнитной решетки и понятие элементарной магнитной ячейки оказываются ненужными, если указать волновые векторы магнитной структуры, а точнее, канал перехода.

В случае нелифшицевских звезд соотношение (4) приводит к модулированным магнитным структурам. Действительно, в кристаллах с центром инверсии любая нелифшицевская звезда наряду с лучом $\mathbf{k}$ содержит и луч $\mathbf{- k}$, так что всегда имеет место двухлучевой канал, в котором соотношение (4) принимает вид

$$
\mathbf{S}_{n i}=e^{i \mathbf{k}_{L} \mathbf{t}_{n}} \mathbf{S}_{0 i}^{\mathbf{k}}+e^{-i \mathbf{k}_{L} \mathbf{t}_{n}} \mathbf{S}_{0 i}^{\mathbf{k}} \text {. }
$$

Это соотношение описывает структуру типа волны спиновой плотности (продольной или поперечной), если лучевой вклад $\mathbf{S}_{0 i}^{\mathbf{k}}=S e^{i \theta} \mathrm{m}$, где $\mathrm{m}-$ вещественный фактор, а фаза $\theta$ описывает "скользящую" степень свободы этой волны, и некоторую спиральную структуру, если $\mathbf{S}_{0 i}^{\mathbf{k}}=S\left(\mathbf{m}_{1 i}+i \mathbf{m}_{2 i}\right)$, где $\mathbf{m}_{1 i}$ и $\mathbf{m}_{2 i}$ - два ортогональных вектора, перпендикулярных волновому вектору $\mathbf{k}$.

Итак, мы приходим к выводу, что для полного задания любой магнитной структуры кристалла нет необходимости указывать отдельно вектор спина на каждом 
магнитном атоме кристалла. Достаточно задать канал перехода (т. е. участвующие лучи звезды) и набор лучевых вкладов - векторов $\mathbf{S}_{0 i}^{L}$, задаваемых в нулевой примитивной ячейке кристалла. Если канал и лучевые вклады известны, соотношение (4) позволяет найти векторы спина атомов в любой ячейке кристалла. Максимальное число векторных параметров $\mathbf{S}_{0 i}^{L}$ равно $l_{\mathbf{k}} \sigma$, где $\sigma$ - число магнитных атомов в примитивной ячейке кристалла.

В основе фундаментального соотношения (4) лежит гипотеза, что магнитная структура описывается одной звездой волнового вектора. Это утверждение является обобщением огромного экспериментального материала по магнитным структурам и имеет также теоретическое обоснование в рамках теории фазовых переходов Ландау. Есть несколько исключений, когда структура характеризуется сразу двумя звездами. Каждая из них обычно характеризует упорядочение ортогональных друг другу проекций атомного магнитного момента. Примером может служить ферромагнитная спираль, обнаруженная в Но и Er. В этом случае геликоидальное упорядочение проекций спинов на базисную плоскость характеризуется нелифшицевской звездой, а ферромагнитная составляющая спинов на гексагональную ось - звездой $\mathbf{k}=0$.

\section{2. Описание магнитных структур с помощью неприводимых пред-} ставлений пространственных групп. Дальнейшего углубления описания магнитной структуры можно достичь, разложив лучевые вклады по базисным функциям неприводимых представлений группы волнового вектора $\mathbf{k}_{L}$. Это разложение можно записать в виде

$$
\mathbf{S}_{0 i}^{L}=\sum_{\nu} \sum_{\lambda} C_{\lambda}^{L \nu} \mathbf{S}\left(\mathbf{k}_{\lambda} \nu \mid i\right)
$$

где $\mathbf{S}\left({ }_{\lambda}^{\mathbf{k}_{L} \nu} \mid i\right)$ - атомная компонента базисной функции $\nu$-го неприводимого представления группы $G_{\mathbf{k}}$ (неприводимое представление группы $G_{\mathbf{k}}$ будем обозначать $d^{\mathbf{k} \nu}$, индекс $\lambda$ нумерует базисные функции этого представления). Сама базисная функция представляет собой многомерный столбец, составленный из всех атомных компонент кристалла $\mathbf{S}\left({ }_{\lambda}^{\mathbf{k}_{L} \nu} \mid i\right)$, однако благодаря соотношению

$$
\mathbf{S}\left({ }_{\lambda}^{\mathbf{k}_{L} \nu} \mid n i\right)=e^{i \mathbf{k} \mathbf{t}_{n}} \mathbf{S}\left({ }_{\lambda}^{\mathbf{k}_{L} \nu} \mid 0 i\right)
$$

она полностью задается атомными компонентами $\mathbf{S}\left({ }_{\lambda}^{\mathbf{k}_{L} \nu} \mid 0 i\right)$ нулевой примитивной ячейки, которые будем обозначать $\mathbf{S}\left({ }_{\lambda}^{\mathbf{k}_{L} \nu} \mid i\right)$.

Если обозначить многокомпонентный столбец векторов атомных спинов $\mathbf{S}_{n i}$ через $\mathbf{S}^{\{\mathbf{k}\}}$, то благодаря соотношениям (4), (6) и (7) получим следующее представление магнитной структуры $\mathbf{S}^{\{\mathbf{k}\}}$ через базисные функции $\psi_{\lambda}^{\mathbf{k} \nu}$ группы волнового вектора:

$$
\mathbf{S}^{\{\mathbf{k}\}}=\sum_{L \nu} \sum_{\lambda} C_{\lambda}^{L \nu} \psi_{\lambda}^{\mathbf{k}_{L} \nu}
$$

Набор базисных функций для всех лучей звезды осуществляет неприводимое представление пространственной группы кристалла $G$, таким образом, соотношение (8) выражает разложение магнитной структуры по базисным функциям неприводимого представления пространственной группы. Такого рода соотношения лежат в основе теории фазовых переходов Ландау. Соотношение (6) и эквивалентное ему (8) являются точными. Если использовать конструктивную идею Ландау, что фазовый 
переход (в данном случае из парамагнитной в магнитоупорядоченную фазу) идет по одному неприводимому представлению (скажем, $d^{\mathbf{k} \nu}$ ), то в выражениях (6) и (8) сумму по $\nu$ можно опустить, и тогда (6) запишется в виде

$$
\mathbf{S}_{0 i}^{L}=\sum_{\lambda} C_{\lambda}^{L \nu} \mathbf{S}\left(\mathbf{k}_{\lambda} \nu \mid i\right) .
$$

Гипотеза одного неприводимого представления находит свое микроскопическое обоснование в структурных переходах дисторсионного типа, проходящих через механизм мягкой моды. Применительно к магнитным фазовым переходам ее справедливость не столь очевидна, поскольку в парамагнитной фазе кристалла нет никаких мягких магнитных мод, которые ниже температуры магнитного перехода $T_{k}$ могли бы "вмерзать" в кристалл, образуя его магнитную структуру. Однако вблизи $T_{k}$ в системе появляются флуктуации магнитного порядка, которые могут быть классифицированы по неприводимым представлениям пространственной группы кристалла. Те из них, собственная энергия которых раньше (при более высокой температуре) обращается в нуль, как бы вмерзают в кристалл, создавая тем самым его магнитную структуру. Назовем соответствующее неприводимое представление ответственным или релевантным.

Эти рассуждения дают некоторые основания ожидать, что магнитный фазовый переход происходит по одному неприводимому представлению группы $G$ и, следовательно, магнитная структура кристалла должна описываться одним неприводимым представлением.

Подведем теперь итоги. Магнитная структура может быть описана с помощью двух соотношений - (4) и (9). Первое из них определяет трансляционные свойства и сводит магнитную структуру всего кристалла к магнитной структуре его примитивной ячейки. Второе соотношение выражает магнитную структуру примитивной ячейки через коэффициент смешивания базисных функций ответственного представления.

Дифракция определяется известным выражением для сечения упругого магнитного рассеяния [4]

$$
\frac{d \sigma_{\kappa}^{\mathrm{m}}}{d \Omega}=\left(r_{0} \gamma\right)^{2}|\mathbf{M}(\boldsymbol{\kappa})|^{2}
$$

где $r_{0}=e^{2} /\left(m c^{2}\right)$ - электромагнитный радиус электрона, $\gamma=-1.99$ - магнитный момент нейтрона в ядерных магнетонах, a

$$
\mathbf{M}(\boldsymbol{\kappa})=\sum_{l} e^{-i \boldsymbol{\kappa} \mathbf{R}_{l}} F_{l}(\boldsymbol{\kappa})\left(\mathbf{S}_{l}-\left(\mathbf{e} \mathbf{S}_{l}\right) \mathbf{e}\right)
$$

здесь $\mathbf{S}_{l}$ и $F_{l}(\boldsymbol{\kappa})$ - спин и магнитный формфактор $l$-го атома, расположенного в узле решетки $\mathbf{R}_{l}, \mathbf{e}=\boldsymbol{\kappa} / \kappa-$ единичный вектор рассеяния.

В случае, когда в кристалле имеется магнитная структура, характеризующаяся звездой волнового вектора $\left\{\mathbf{k}_{L}\right\}$, спин атома $l\left(\mathbf{R}_{l}=\mathbf{t}_{n}+\mathbf{x}_{i}\right)$ произвольной ячейки кристалла выражается через лучевые вклады $\mathbf{S}_{0 i}^{L}$ посредством соотношения (4). Подставляя его в (11) и суммируя по всем трансляциям $\mathbf{t}_{n}$, представим сечение $d \sigma_{\kappa}^{m} / d \Omega$ в следующем виде:

$$
\frac{1}{N} \frac{d \sigma_{\kappa}^{\mathrm{m}}}{d \Omega}=\left(r_{0} \gamma\right)^{2} \sum_{L} \mathbf{M}_{\boldsymbol{\kappa}}^{L^{*}} \mathbf{M}_{\boldsymbol{\kappa}}^{L} N \sum_{\mathbf{b}} \delta_{\boldsymbol{\kappa}-\mathbf{k}_{L}, \mathbf{b}}
$$


где

$$
\begin{gathered}
\mathbf{M}_{\boldsymbol{\kappa}}^{L}=\mathbf{f}_{\boldsymbol{\kappa}}^{L}-\left(\mathbf{e f}_{\boldsymbol{\kappa}}^{L}\right) \mathbf{e} \\
\mathbf{f}_{\boldsymbol{\kappa}}^{L}=\sum_{i} e^{-i \boldsymbol{\kappa} \mathbf{x}_{i}} F_{i}(\boldsymbol{\kappa}) \mathbf{S}_{0 i}^{L}
\end{gathered}
$$

a суммирование по b ведется по векторам обратной решетки исходного кристалла.

При получении выражения (12) было использовано известное тождество

$$
\frac{1}{N} \sum_{n} e^{-i \mathbf{q} \mathbf{t}_{n}}=\sum_{\mathbf{b}} \delta_{\mathbf{q}, \mathbf{b}}
$$

$\left(N\right.$ - число ячеек в кристалле, $\delta_{\mathbf{q}, \mathbf{b}}-$ символ Кронекера), а также тот факт, что два луча звезды $\mathbf{k}_{L}$ и $\mathbf{k}_{L}^{\prime}$ не могут отличаться на целый вектор обратной решетки. Благодаря последнему обстоятельству рассеяние на магнитной решетке аддитивно по лучевым вкладам и группируется в брэгговские пики, угловое положение которых определяется условиями

$$
\kappa=\mathbf{b}+\mathbf{k}_{L} .
$$

Сечение ядерного рассеяния описывается выражением [4]

$$
\frac{1}{N} \frac{d \sigma_{\kappa}^{\text {яд }}}{d \Omega}=\left|f_{\kappa}^{\text {яд }}\right|^{2} N \sum_{\mathbf{b}} \delta_{\boldsymbol{\kappa}, \mathbf{b}},
$$

означающим появление ядерного пика при условии

$$
\kappa=\mathbf{b}
$$

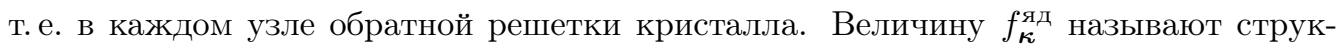
турной амплитудой ядерного рассеяния, а величину $\mathbf{f}_{\kappa}^{L}(14)$ следует назвать структурной амплитудой магнитного рассеяния.

Таким образом, в общем случае пики магнитного рассеяния не совпадают с пиками ядерного рассеяния, и только если магнитная структура характеризуется волновым вектором $\mathbf{k}=0$ (а это имеет место в случае ферромагнитной структуры или антиферромагнитной при совпадении магнитной и кристаллической ячеек), они налагаются друг на друга. Наблюдение системы магнитных рефлексов позволяет определить по соотношению (16) волновые векторы магнитной структуры, что составляет первый этап нейтронографического исследования магнитной структуры. Измерение интенсивностей пиков в силу соотношений (12)-(14) позволяет определить векторы атомных спинов $\mathbf{S}_{0 i}$, что составляет второй, окончательный этап нейтронографического исследования. Рассмотрим теперь оба эти этапа более подробно.

Из соотношения (16) следует, что каждый луч звезды порождает систему магнитных рефлексов, размноженных из некоторого данного всеми трансляциями обратной решетки исходного кристалла. Каждый магнитный рефлекс, характеризуемый вектором рассеяния $\boldsymbol{\kappa}\left(\boldsymbol{\kappa}=h \mathbf{b}_{1}+k \mathbf{b}_{2}+l \mathbf{b}_{3}\right)$, может быть изображен тройкой чисел $(h, k, l)$, называемых миллеровскими индексами. Если из миллеровских индексов магнитных рефлексов вычесть индексы ядерных рефлексов, то будут получены миллеровские индексы $\left(h_{L}, k_{L}, l_{L}\right)$ луча $\mathbf{k}_{L}$ исследуемой магнитной структуры. Так должны определяться волновые векторы магнитной структуры, составляющие канал перехода. 
Из этого примера ясно видно, каким образом канал перехода должен определяться по системе магнитных рефлексов. В работах [21], [22] составлена таблица, по которой для наблюдаемой системы магнитных рефлексов можно определить канал перехода и магнитную решетку (ее элементарную ячейку и символ шубниковской группы) для всех лифшицевских звезд всех 14 исходных решеток Браве.

Очевидно, что канал перехода в принципе может определяться по системе магнитных рефлексов (т. е. без рассмотрения интенсивностей) только из нейтронографии на монокристаллах. При нейтронографии на порошках следует иметь в виду, что, например, для кубического кристалла рефлексы (100), (010) и (001) неразличимы, и для установления канала перехода, т. е. магнитной решетки, необходимо сравнение интенсивностей магнитных рефлексов. Таким образом, исследование порошков позволяет по системе магнитных рефлексов установить только звезду волнового вектора, так что магнитную структуру следует представить в виде суперпозиции (4) спиновых функций на отдельных лучах и подбирать коэффициенты межлучевого смешивания (или лучевые вклады $\mathbf{S}_{0 i}^{L}$ ) из наилучшего совпадения с экспериментом.

После того как выявлена звезда волнового вектора и канал перехода, необходимо определить векторы атомных спинов в примитивной ячейке, для чего следует воспользоваться выражением для интенсивности магнитных рефлексов

$$
I_{\kappa}^{L} \sim \mathbf{M}_{\kappa}^{L^{*}} \mathbf{M}_{\kappa}^{L}
$$

Каждый рефлекс из всей серии магнитных рефлексов, порождаемых одним лучом $\mathbf{k}_{L}$, содержит $\sigma$ комплексных векторов $\mathbf{S}_{0 i}^{L}$, которые можно найти вариационной процедурой из наилучшего согласия с измеренными интенсивностями. Аналогичная процедура для серии рефлексов, отвечающих другому лучу, позволяет найти векторы соответствующего лучевого вклада и т. д. Каждый раз варьируются $\sigma$ комплексных векторов, если исследования ведутся на монокристалле (однодоме́нном), и $l_{\mathbf{k}} \sigma$ векторов в случае исследований на порошковой нейтронограмме.

Это соответствует традиционному пути расшифровки магнитной структуры, когда ищут непосредственно векторы атомных спинов (или лучевых вкладов $\mathbf{S}_{0 i}^{L}$ в случае многолучевой магнитной структуры). Используя теорию представлений пространственных групп, можно число варьируемых параметров резко сократить. Действительно, подставим выражение (9) для лучевого вклада через базисные функции некоторого неприводимого представления группы волнового вектора $G_{\mathbf{k}}$ в формулу (14) для структурной магнитной амплитуды. Мы видим, что она разлагается на сумму нормальных амплитуд $\mathbf{f}_{\boldsymbol{\kappa} \lambda}^{L \nu}$ :

$$
\mathbf{f}_{\boldsymbol{\kappa}}^{L}=\sum_{\lambda} C_{\lambda}^{L \nu} \sum_{i} e^{-i \boldsymbol{\kappa} \mathbf{x}_{i}} F_{i}(\boldsymbol{\kappa}) \mathbf{S}\left({ }_{\lambda}^{\mathbf{k}_{L} \nu} \mid 0 i\right) \equiv \sum_{\lambda} C_{\lambda}^{L \nu} \mathbf{f}_{\boldsymbol{\kappa} \lambda}^{L \nu},
$$

которые могут быть вычислены заранее и поэтому считаются известными. Таким образом, при исследовании выражения (19) в формуле (18) для интенсивности брэгговского пика варьированию подлежат не $\sigma$ неизвестных комплексных векторов $\mathbf{S}_{0 i}^{L}$, a $l_{\nu}$ коэффициентов смешивания $C_{\lambda}^{L \nu}$, где $l_{\nu}$ - небольшие числа $(1,2,3$, реже 6$)$. Сокращение переменных варьирования в подгоночной процедуре особенно эффективно при большом числе магнитных атомов $\sigma$ в примитивной ячейке кристалла.

В исследуемой магнитной структуре ответственное неприводимое представление заранее неизвестно, поэтому следует перебирать одно за другим все неприводимые 
представления пространственной группы с заданной звездой (их число невелико, и сами неприводимые представления берутся из справочников). Каждый раз при этом приходится иметь дело с небольшим числом варьируемых параметров - коэффициентов смешивания. То представление, для которого получается наилучшее согласие рассчитанных и наблюдаемых интенсивностей, и определяет ответственное представление. Если окажется, что ни одно неприводимое представление не дает удовлетворительного согласия, следует комбинировать их, предполагая, что магнитная структура описывается приводимым представлением пространственной группы.

Выше было показано, что проблема описания и расшифровки магнитной структуры кристалла сводится к вычислению базисных функций неприводимых представлений его пространственной группы, т. е. векторных величин $\mathbf{S}\left(\begin{array}{c}\mathbf{k} \nu \\ \lambda\end{array} i\right)$. Покажем, каким образом они должны вычисляться.

Представление пространственной группы $D^{\{\mathbf{k}\} \nu}$ строится из представлений $d^{\mathbf{k} \nu}$ группы волнового вектора $G_{\mathbf{k}}$. Группа волнового вектора является подгруппой пространственной группы $G$, составленной из элементов $g$, оставляющих волновой вектор $\mathbf{k}$ инвариантным, т. е. элемент $g$ входит в $G_{\mathbf{k}}$, если

$$
g \mathbf{k}=\mathbf{k}+\mathbf{b} .
$$

Представлением группы $G_{\mathbf{k}}$ является набор матриц $d_{\lambda \mu}^{\mathbf{k} \nu}(g)$, сопоставленных каждому элементу $g \in G_{\mathbf{k}}$ и удовлетворяющих тем же правилам умножения, что и сами элементы группы. Матрицы неприводимых представлений затабулированы для элементов $g=\left\{h \mid \boldsymbol{\tau}_{h}\right\}$ так называемого нулевого блока группы $G_{\mathbf{k}}$, т. е. для совокупности элементов, не содержащих целых трансляций $\mathbf{t}\left(\boldsymbol{\tau}_{h}-\right.$ некоторая дробная часть трансляции, сопровождающая поворот $h$ ). Для произвольных элементов группы $G_{\mathbf{k}}$ имеет место соотношение

$$
d_{\lambda \mu}^{\mathbf{k} \nu}\left(\left\{h \mid \boldsymbol{\tau}_{h}+\mathbf{t}\right\}\right)=e^{-i \mathbf{k} \mathbf{t}_{n}} d_{\lambda \mu}^{\mathbf{k} \nu}\left(\left\{h \mid \boldsymbol{\tau}_{h}\right\}\right),
$$

сводящее все к элементам нулевого блока. Матрица $d_{\lambda \mu}^{\mathbf{k} \nu}(g)$ определена на некотором базисе функций $\psi_{\lambda}^{\mathbf{k} \nu}$, преобразующихся друг через друга под действием элемента $g \in G_{\mathbf{k}}$ посредством соотношения

$$
T(g) \psi_{\lambda}^{\mathbf{k} \nu}=\sum_{\mu} d_{\mu \lambda}^{\mathbf{k} \nu}(g) \psi_{\mu}^{\mathbf{k} \nu}
$$

Если подействовать на функцию $\psi_{\lambda}^{\mathbf{k} \nu}$ элементом $g$ пространственной группы $G$, не принадлежащим $G_{\mathbf{k}}$, будут возникать линейные комбинации, составленные из функций $\psi_{\lambda}^{\mathbf{k}_{L} \nu}$, принадлежащих другим лучам звезды $\{\mathbf{k}\}$. Таким образом, набор функций $\psi_{\lambda}^{\mathbf{k}_{L} \nu}$ с номерами $L=1,2, \ldots, l_{\mathbf{k}}$ и $\lambda=1,2, \ldots, l_{\nu}$ преобразуется друг через друга, осуществляя неприводимое представление группы $G$ с тем же номером $\nu$, что и неприводимое представление группы $G_{\mathbf{k}}$. Размерность представления, очевидно, равна $l_{\mathbf{k}} \times l_{\nu}$. В дальнейшем все окончательные формулы будут содержать лишь матрицы неприводимых представлений группы $G_{\mathbf{k}}$, поэтому мы не поясняем структуры матриц представления большой группы $G$.

Для вычисления базисных функций неприводимых представлений групп $G$ и $G_{\mathbf{k}}$, по которым возможно разложение спиновой плотности кристалла в виде (6) или (8) 
(а также других величин, например плотности заряда, плотности дипольного момента и т. п.), полезно использовать приводимые представления этих групп, построенные на локализованных атомных функциях, тем самым включающих информацию об интересующих нас свойствах кристалла. Идея построения таких представлений состоит в следующем.

Пусть состояние каждого атома характеризуется некоторой атомной функцией, локализованной вблизи его равновесного положения в кристалле. Состояние кристалла в целом характеризуется набором таких функций, заданных для отдельных атомов и образующих некоторый многомерный вектор в пространстве атомных функций. Под действием элементов пространственной группы этот набор будет переводиться в другой набор атомных функций из того же пространства, реализуя тем самым некоторое представление этой группы, которое в общем случае должно быть приводимым. Свойства этого представления зависят не только от структуры кристалла, т. е. от расположения атомов в пространстве, но и от физического содержания взятых атомных функций.

Атомная функция может не описывать никакой специфической характеристики атома, кроме лишь его определенного номера в кристалле, так что при действии элементов пространственной группы на вектор такого типа состояния кристалла все сводится к перестановке атомных номеров. В другом случае с каждым атомом может быть связана характеристика, описываемая полярным вектором (например, смещением его из положения равновесия) или аксиальным вектором (псевдовектором), которым может быть магнитный момент атома. При действии элементов группы $G$ вместе с изменением номера атома будет преобразовываться и приписываемый ему вектор. Представления пространственной группы, порождаемые на базисе скалярных, векторных и псевдовекторных атомных функций, называются соответственно перестановочным, механическим и магнитным. Механическое представление используется для описания фононов в кристалле и атомных смещений при структурных фазовых переходах, магнитное - для описания магнитных структур кристаллов, перестановочное представление носит вспомогательный характер и будет использовано нами для анализа обменного гамильтониана в кристалле.

Приведем окончательный вид матриц этих трех представлений, вывод которых в столь удобной форме сделан в работах [21], [23]. Перестановочное, механическое и магнитное представления группы волнового вектора $G_{\mathbf{k}}$ для заданного кристалла будем обозначать соответственно $d_{\mathrm{p}}^{\mathbf{k}}, d_{\mathrm{m}}^{\mathbf{k}}$ и $d_{\mathrm{M}}^{\mathbf{k}}$. Их матрицы имеют следующий вид:

$$
\begin{aligned}
\left\{d_{\mathrm{p}}^{\mathbf{k}}(g)\right\}_{i j} & =e^{-i \mathbf{k a}_{\mathrm{p}}(g, j)} \delta_{i, g j}, \\
\left\{d_{\mathrm{m}}^{\mathbf{k}}(g)\right\}_{i \alpha, j \beta} & =e^{-i \mathbf{k a}_{\mathrm{p}}(g, j)} \delta_{i, g j} R_{h}^{\alpha \beta}, \\
\left\{d_{\mathrm{M}}^{\mathbf{k}}(g)\right\}_{i \alpha, j \beta} & =e^{-i \mathbf{k a}_{\mathrm{p}}(g, j)} \delta_{i, g j} \delta_{h} R_{h}^{\alpha \beta} .
\end{aligned}
$$

Здесь $i$ и $j$ - номера атомов в примитивной ячейке кристалла, $\alpha$ и $\beta$ - векторные индексы $x, y, z$. Таким образом, матрицы перестановочного представления имеют размерность $\sigma \times \sigma$, а векторных представлений $-3 \sigma \times 3 \sigma . R_{h}^{\alpha \beta}$ - матрица поворота радиус-вектора под действием поворотной части $h$ элемента $g=\left\{h \mid \boldsymbol{\tau}_{h}\right\}$, а $\delta_{h}= \pm 1$ в зависимости от того, является ли элемент $h$ элементом первого рода (обычные повороты) или второго рода (инверсия, отражение). Вектор $\mathbf{a}_{\mathrm{p}}(g, j)$, называемый возвращающей трансляиией, указывает, в какую ячейку переводится атом номера $j$

7 Теоретическая и математическая физика, т. 168, № 3, 2011 г. 
из нулевой ячейки под действием элемента $g$ (в новой ячейке он имеет номер $i$ ), и определяется из соотношения

$$
g \mathbf{x}_{j}=h \mathbf{x}_{i}+\boldsymbol{\tau}_{h} \equiv \mathbf{x}_{i}+\mathbf{a}_{\mathbf{p}}(g, j)
$$

Таким образом, матрицы всех трех представлений могут быть легко вычислены, если составить таблицу перестановок атомов примитивной ячейки под действием элементов группы $G_{\mathbf{k}}$.

Введенные представления могут быть разложены по неприводимым представлениям группы $G_{\mathbf{k}}$. Например, для перестановочного представления это разложение имеет вид

$$
d_{\mathrm{p}}^{\mathbf{k}}=\sum_{\nu} n_{\mathrm{p}}^{\nu} d^{\mathbf{k} \nu}
$$

где

$$
n_{\mathrm{p}}^{\nu}=\frac{1}{n\left(G_{\mathbf{k}}^{0}\right)} \sum_{h \in G_{\mathbf{k}}^{0}} \chi_{\mathrm{p}}^{\mathbf{k}}(g) \chi^{\mathbf{k} \nu^{*}}(g)
$$

здесь $\chi^{\mathbf{k} \nu}(g)$ - характер неприводимого представления $d^{\mathbf{k} \nu}$, a $\chi_{\mathrm{p}}^{\mathbf{k}}$ - характер перестановочного представления,

$$
\chi_{\mathrm{p}}^{\mathbf{k}}(g)=\sum_{j} e^{-i \mathbf{k} \mathbf{a}_{\mathrm{p}}(g, j)} \delta_{j, g j} .
$$

Суммирование в выражении (28) ведется по элементам нулевого блока группы $G_{\mathbf{k}}$, $n\left(G_{\mathbf{k}}\right)$ - число этих элементов (совпадает с числом элементов точечной группы $G_{\mathbf{k}}^{0}$, соответствующей группе $G_{\mathbf{k}}$ ). Для механического и магнитного представлений могут быть получены формулы, аналогичные (27), (28), а характеры их даются формулами

$$
\begin{aligned}
\chi_{\mathrm{m}}^{\mathbf{k}}(g) & =\chi_{\mathrm{p}}^{\mathbf{k}}(g) \operatorname{Sp} R_{h}, \\
\chi_{\mathrm{M}}^{\mathbf{k}}(g) & =\chi_{\mathrm{p}}^{\mathbf{k}}(g)\left(\delta_{h} \operatorname{Sp} R_{h}\right) .
\end{aligned}
$$

$\operatorname{Sp} R_{h}$ можно рассматривать как характер представления $V$, по которому преобразуется вектор под действием поворотной части элемента пространственной группы, a $\delta_{h} \mathrm{Sp} R_{h}$ - характер представления $V^{\prime}$, по которому преобразуется псевдовектор. Из соотношений (30) и (31) поэтому следует, что векторные представления $d_{\mathrm{m}}^{\mathbf{k}}$ и $d_{\mathrm{M}}^{\mathbf{k}}$ являются прямым произведением перестановочного представления $d_{\mathrm{p}}^{\mathbf{k}}$ на представление $V$ и $V^{\prime}$, т. е.

$$
d_{\mathrm{m}}^{\mathbf{k}}=d_{\mathrm{p}}^{\mathbf{k}} \times V, \quad d_{\mathrm{M}}^{\mathbf{k}}=d_{\mathrm{p}}^{\mathbf{k}} \times V^{\prime} .
$$

Для построения базисных функций $\psi_{\lambda}^{\mathbf{k} \nu}$ неприводимых представлений группы $G_{\mathbf{k}}$ можно воспользоваться общей формулой оператора проектирования

$$
\psi_{\lambda}^{\mathbf{k} \nu}=\frac{1}{N} \sum_{g \in G_{\mathbf{k}}} d_{\lambda \mu}^{\mathbf{k} \nu^{*}}(g) T(g) \psi
$$

где $d_{\lambda \mu}^{\mathbf{k} \nu}$ - матрицы выбранного представления, а $\psi$ - некоторая стартовая функция. Если фиксировать индекс $\mu$, формула (33) определяет $l_{\nu}$ базисных функций 
$l_{\nu}$-мерного неприводимого представления $d^{\mathbf{k}} \nu$. Выбирая в качестве стартовой функции вектор состояния в пространстве локализованных атомных функций, получим один из трех типов базисных функций - скалярный, векторный или псевдовекторный.

Приведем окончательные формулы для атомных компонент функций скалярного, векторного и псевдовекторного базисов соответственно:

$$
\begin{aligned}
\varphi\left({ }_{\lambda}^{\mathbf{k} \nu} \mid i\right) & =\sum_{g \in G_{\mathbf{k}}^{0}} d_{\lambda[\mu]}^{\mathbf{k} \nu}{ }^{*}(g) e^{-i \mathbf{k a}_{\mathrm{p}}(g, j)} \delta_{i, g[j]}, \\
u^{\alpha}\left({ }_{\lambda}^{\mathbf{k} \nu} \mid i\right) & =\sum_{g \in G_{\mathbf{k}}^{0}} d_{\lambda[\mu]}^{\mathbf{k} \nu}{ }^{*}(g) e^{-i \mathbf{k a}_{\mathrm{p}}(g, j)} \delta_{i, g[j]} R_{h}^{\alpha[\beta]}, \\
S^{\alpha}\left({ }_{\lambda}^{\mathbf{k} \nu} \mid i\right) & =\sum_{g \in G_{\mathbf{k}}^{0}} d_{\lambda[\mu]}^{\mathbf{k} \nu}{ }^{*}(g) e^{-i \mathbf{k a}_{\mathrm{p}}(g, j)} \delta_{i, g[j]} \delta_{h} R_{h}^{\alpha[\beta]} .
\end{aligned}
$$

Векторы $\mathbf{S}(\underset{\lambda}{\mathbf{k} \nu} \mid i)$ являются именно теми величинами, которые фигурировали в разложении спиновой плотности кристалла (6) (и (9)). Индексы $\mu, j$ и $\beta$, заключенные в квадратные скобки, должны при вычислениях фиксироваться. Переход к другому набору индексов означает смену стартовой функции в формуле (33) и может либо привести к тождественному нулю, либо дать новый набор функций, преобразующихся по данному представлению, если оно входит в состав соответствующего приводимого представления $d_{\mathrm{p}}^{\mathbf{k}}, d_{m}^{\mathbf{k}}$ или $d_{\mathrm{M}}^{\mathbf{k}}$ не один раз.

Базисные функции для другого луча $\mathbf{k}_{L}$ звезды волнового вектора могут быть получены действием соответствующего элемента $g_{L}=\left\{h_{L} \mid \boldsymbol{\tau}_{h_{L}}\right\}$ на базисную функцию первого луча $\mathbf{k}$. Для псевдовекторного базиса, например, соответствующая формула имеет вид

$$
S^{\alpha}\left(\mathbf{k}_{\lambda} \nu \mid i^{\prime}\right)=e^{-i \mathbf{k}_{L} \mathbf{a}_{\mathrm{p}}\left(g_{L}, j\right)} \delta_{h_{L}} \sum_{\beta} R_{h}^{\alpha \beta} S^{\beta}(\underset{\lambda}{\mathbf{k} \nu} \mid i) .
$$

Смена номеров атомов $i$ и $i^{\prime}$ происходит согласно правилу

$$
g_{L} \mathbf{x}_{i}=h \mathbf{x}_{i}+\boldsymbol{\tau}_{h_{L}} \equiv \mathbf{x}_{i^{\prime}}+\mathbf{a}_{\mathrm{p}}\left(g_{L}, i\right) .
$$

Для фактического вычисления псевдовекторных базисных функций достаточно использовать формулы (36) и (37). Заметим, что необходимо вычислять базисные функции только тех неприводимых представлений, которые входят в состав магнитного представления $d_{\mathrm{M}}^{\mathbf{k}}$ (для других неприводимых представлений формулы (36) и (37) должны давать тождественный нуль).

\section{3. МАЛОУГЛОВОЕ МНОГОКРАТНОЕ РАССЕЯНИЕ}

Малоугловое рассеяние - один из принципиальных методов для исследования крупномасштабных неоднородностей в конденсированной материи. При пропускании узкого коллимированного пучка рентгеновских лучей или медленных нейтронов через мелко раздробленный материал наблюдается расходимость этого пучка, т. е. его ширина возрастает. Это явление приписывается дифракции (отклонению падающего пучка) и рефракции (преломлению падающего пучка при прохождении 
через неоднородность) на отдельных частицах вещества с линейными размерами на несколько порядков бо́льшими длины волны падающих нейтронов. Наблюдаемое уширение пучка следует отличать от когерентных дифракционных спектров, определяющих структуру кристалла внутри крупномасштабных неоднородностей с межплоскостными расстояниями порядка длины волны.

Для введения понятий теории малоуглового рассеяния удобно сначала рассмотреть амплитуду рассеяния сферической прямоугольной потенциальной ямой глубиной $U$ и радиуса $R$. Предположим, что $U \ll E$, где $E-$ энергия налетающих частиц. Выполнение этого условия означает, что рассеяние быстрых частиц является малоугловым. В эйкональном приближении [24] амплитуда рассеяния принимает вид

$$
f(q)=-i k \int\left\{e^{2 i \delta(\rho)}-1\right\} J_{0}(q \rho) \rho d \rho,
$$

где $k$ - импульс налетающих частиц, $q=2 k \sin (\theta / 2) \approx k \theta$ - переданный импульс, выраженный через угол рассеяния $\theta$. В квазиклассическом приближении фазовый сдвиг $\delta(\rho)$ дается выражением

$$
\delta(\rho)=-\frac{m}{2 k \hbar^{2}} \int_{-\infty}^{\infty} U\left(\sqrt{z^{2}+\rho^{2}}\right) d z .
$$

Для сферической неоднородности имеем

$$
U(r)=-U \theta(R-r)
$$

где $\theta(R>r)=1, \theta(R<r)=0$ - ступенька.

Подставляя уравнение (41) в уравнения (40) и (39), получаем

$$
f(q)=-i k R^{2} \int_{0}^{1}\left\{e^{i \alpha \sqrt{1-x^{2}}}-1\right\} J_{0}(q R x) x d x
$$

где $\alpha=(U / E) k R$ - “борновский" параметр. В нейтронной оптике этот параметр выражается через показатель преломления для нейтронов $n$ следующим образом:

$$
\alpha=\left(1-n^{2}\right) k R \approx \frac{4 \pi}{\lambda}(1-n) R
$$

где во втором равенстве учтена близость показателя преломления к единице и использовано определение $\lambda$ для длины волны нейтрона. Приведем здесь также известное выражение для показателя преломления нейтронов:

$$
n=\left[1-\frac{\lambda^{2} N \bar{b}}{\pi}\right]^{1 / 2} \approx 1-\frac{\lambda^{2} N \bar{b}}{2 \pi},
$$

где $N$ - число ядер в единице объема, а $\bar{b}$ - среднее значение амплитуды когерентного рассеяния для связанных атомов. Величина $\alpha$ имеет смысл разности между сдвигами фазы при прохождении нейтроном неоднородности и при прохождении того же расстояния в вакууме.

Интеграл в уравнении (42) можно представить в общем виде через цилиндрические функции, однако из-за громоздкости здесь мы его не приводим (см. работу [25]). 
Вычисление сечения рассеяния падающего пучка нейтронов упрощается для двух предельных случаев: когда существенна только дифракция $(\alpha \ll 1)$ и когда преобладает рефракция $(\alpha \gg 1)$.

В случае дифракции $(\alpha \ll 1)$ выражение для амплитуды рассеяния сводится к известному борновскому приближению

$$
f(q)=k R^{2} \alpha \sqrt{\frac{\pi}{2}} \frac{J_{3 / 2}(q R)}{(q R)^{3 / 2}} .
$$

Таким образом, в борновском приближении дифференциальное сечение рассеяния $\sigma_{\mathrm{B}}(q)$, определяемое квадратом модуля амплитуды рассеяния, имеет вид

$$
\sigma_{\mathrm{B}}(q)=k^{2} R^{4} \alpha^{2} \frac{\pi}{2} \frac{J_{3 / 2}^{2}(q R)}{(q R)^{3}} .
$$

Как хорошо известно, в борновском приближении в случае больших скоростей нейтронов $k R \gg 1$ (именно этот случай и интересует нас при исследовании рассеяния на малые углы) рассеяние резко анизотропно и направлено вперед в узком конусе с углом раствора

$$
\Delta \theta_{\mathrm{D}} \sim \frac{1}{k R}
$$

Следовательно, увеличение ширины прошедшего пучка, обусловленное дифракцией, пропорционально $\lambda / R$, и этот факт лежит в основе объяснения всех экспериментальных результатов по изучению рассеяния нейтронов в области дифракции $(\alpha \ll 1)$. В частности, впервые формула (47) была количественно подтверждена Вейссом [26] в опытах по рассеянию нейтронов на частицах угольной сажи диаметром около $500 \AA$.

Полное сечение рассеяния $\sigma$ на малые углы находится интегрированием выражения (46) по телесному углу $\Omega$ и в борновском приближении равно

$$
\sigma_{\mathrm{B}}=\frac{1}{2} \pi R^{2} \alpha^{2}
$$

Оно зависит от радиуса неоднородности $R$ и согласно (43) и (44) от длины волны $\lambda$ и амплитуды когерентного рассеяния $\bar{b}$ на атомах неоднородности.

В асимптотическом пределе $q R \gg 1$ дифференциальное сечение (46) убывает как $q^{-4}$. Обобщение этого результата на малоугловое рассеяние нейтронов реальными средами приводит к известному закону Порода $\sigma(q) \propto q^{-n}$, где $n$ - показатель, который может отличаться от 4 в зависимости от типа рассматриваемой неоднородности и ее фрактальных свойств. Например, для поверхностных и объемных фракталов дифференциальное сечение рассеяния уменьшается как $q^{-6+D_{\mathrm{S}}}$ и $q^{-D_{\mathrm{V}}}$ соответственно, $3>D_{\mathrm{V}, \mathrm{S}}>2$. Заметим также, что в случае критических флуктуаций сечение рассеяния уменьшается как $q^{-2+\eta} \approx q^{-2}$.

В случае рефракции $(\alpha \gg 1)$ формула для амплитуды рассеяния приобретает иной вид:

$$
\begin{gathered}
f(q)=-i k R^{2}\left\{\sqrt{\frac{\pi}{2}} \frac{i \alpha}{\left(\alpha^{2}+(q R)^{2}\right)^{3 / 4}} H_{3 / 2}^{(1)}\left(\sqrt{\alpha^{2}+(q R)^{2}}\right)-\right. \\
\left.-\frac{J_{1}(q R)}{q R}-\frac{\alpha}{\left(\alpha^{2}+(q R)^{2}\right)^{3 / 2}} J_{0}(q R)\right\} .
\end{gathered}
$$


В этом случае выражение для дифференциального сечения рассеяния имеет гораздо более сложную форму:

$$
\sigma_{\mathrm{R}}(q)=k^{2} R^{4}\left\{\frac{J_{1}^{2}(q R)}{(q R)^{2}}+\frac{\alpha^{2}}{\left[\alpha^{2}+(q R)^{2}\right]^{2}}-\frac{2 \alpha}{\alpha^{2}+(q R)^{2}} \frac{J_{1}(q R)}{q R} \sin \sqrt{\alpha^{2}+(q R)^{2}}\right\}
$$

Здесь первый член интерпретируется как дифракция нейтронов на неоднородности, второй - как рефракция нейтронов при прохождении через неоднородность, а смешанный третий член представляет собой интерференцию между дифракцией и рефракцией [26]. В отличие от борновского приближения, полностью обусловленного только явлением дифракции и поэтому, естественно, названным областью дифракции, противоположный случай $\alpha \gg 1$ описывается как дифракцией, так и рефракцией, но называется областью рефракции.

Первый и второй члены в выражении (50) дают одинаковые вклады, равные $\pi R^{2}$, в полное сечение рассеяния, тогда как третий член дает нулевой вклад благодаря своему осциллирующему характеру. Поэтому полное сечение рассеяния в рефракционной области имеет следующий вид

$$
\sigma_{\mathrm{R}}=2 \pi R^{2}
$$

т. е. представляет собой удвоенное геометрическое сечение. Смысл этого результата довольно прост. В случае $\alpha \gg 1$ все нейтроны с прицельным расстоянием, меньшим радиуса неоднородности, выбывают из падающего пучка. Неоднородность становится эквивалентной "поглощающему" шару и согласно известному из оптики принципу Бабине полное сечение рассеяния равно удвоенному сечению “поглощения".

Первый член в формуле (50) имеет ширину пучка, пропорциональную $\lambda / R$. При больших $\alpha$ уширение пучка становится преобладающим благодаря второму слагаемому. Нетрудно видеть, что это уширение пучка определяется величиной $q R \sim \alpha$, т. e.

$$
\Delta \theta_{\mathrm{R}} \sim \frac{\alpha}{k R} \sim \lambda^{2} N \bar{b}
$$

Таким образом, увеличение ширины прошедшего пучка, обусловленное рефракцией, пропорционально $\lambda^{2} \bar{b}$, но не зависит от радиуса неоднородности, и теперь уже этот факт лежит в основе объяснения всех экспериментальных результатов по изучению рассеяния нейтронов в области рефракции $(\alpha \gg 1)$. И опять первая экспериментальная проверка этих соотношений была проведена Вейссом [26] при изучении прохождения пучка нейтронов через образец порошкообразного висмута.

Следует заметить, что расширение пучка падающих нейтронов связано не только с рассеянием на отдельных частицах (неоднородностях) вещества, но и с многократным преломлением нейтронов на границах зерен порошка и магнитных доменов. В этом отношении показателен пример многократного преломления на границах доменов ненамагниченного ферромагнетика - железа [27]. Обобщая формулу (44), получим для показателя преломления нейтронов в железе выражение

$$
n^{2}=1-\lambda^{2}\left(\frac{N \bar{b}}{\pi} \pm \mu_{\mathrm{n}} \frac{B}{E}\right) \equiv 1-\lambda^{2}\left(\frac{N \bar{b}}{\pi} \pm \frac{m_{\mathrm{n}} \mu_{\mathrm{n}} B}{2 \pi^{2} \hbar^{2}}\right)
$$


где $\mu_{\mathrm{n}}$ - магнитный момент нейтрона, $m_{\mathrm{n}}$ - его масса, а $B$ - магнитная индукция в домене. Выбор знака зависит от ориентации спина нейтрона относительно направления магнитного момента домена. Нейтрон, проходя в железе из одного домена в другой, испытывает преломление на границах доменов. Отклонение нейтрона зависит от ориентации спина нейтрона, ориентации самой границы и направлений магнитных моментов пограничных доменов. Среднее значение отклонения $\bar{\theta}$ может быть определено в приближении беспорядочной ориентации границ доменов и их намагниченностей [27]. Максимальное возможное отклонение нейтрона равно удвоенному критическому углу $\left(\theta_{\mathrm{c}}=\left(2 \mu_{\mathrm{n}} B / E\right)^{1 / 2}\right)$, ниже которого имеет место полное отражение, однако среднее отклонение гораздо меньше. Отметим, что в намагниченном до насыщения образце различие в показателях преломления отсутствует и никакого преломления не происходит. Установлено теоретически и подтверждено экспериментально [27], что в первоначально параллельном пучке нейтронов при пропускании его через образец из ненамагниченного железа установится гауссово угловое распределение с шириной, определяемой соотношением

$$
\Delta \theta_{\mathrm{Fe}}=\bar{\theta}\left(\frac{L}{\delta}\right)^{1 / 2},
$$

где $L$ - толщина образца, а $\delta$ - размер домена.

Очевидно, что в случае, когда толщина образца $L$ превышает длину свободного пробега $l_{R}$, имеет место многократное рассеяние. Теория многократного рассеяния для атомных столкновений, известная как теория Мольера, для дифракционной области была применена в работе [28], а для рефракционной области - в работе [29].

В качестве примера изучим систему сферических частиц или пор радиуса $R$, случайно распределенных по объему образца $V$, в сильно разбавленном случае, когда объем $\delta V$ образца, занятый частицами, мал по сравнению с $V$.

В дифракционной области длина свободного пробега $l$, определяемая полным сечением рассеяния и плотностью частиц согласно известной формуле, имеет вид

$$
l_{\mathrm{B}}=\frac{4 \pi R^{3}}{3 \sigma_{B}} \frac{V}{\delta V}=\frac{8 R}{3 \alpha^{2}} \frac{V}{\delta V} .
$$

Заметим, что в этом случае соотношение $l_{\mathrm{B}} \sim \alpha^{-2} \gg R$ выполняется для произвольной концентрации частиц.

Соответственно длина свободного пробега в рефракционной области дается формулой

$$
l_{\mathrm{R}}=\frac{2 R}{3} \frac{V}{\delta V} .
$$

Легко видеть, что теперь $l_{\mathrm{R}} \gg R$ только в случае, когда $\delta V \ll V$. В этом и заключается причина упомянутого выше ограничения рассмотрением сильно разбавленного случая.

Было показано [29], что существуют две области для толщины образца $L$ с различным поведением многократного рассеяния нейтронов в каждой области. В области малых значений толщины $L \ll l \alpha^{2} \ln \alpha=L_{0}$ интенсивность рассеянных частиц $I(q)$ дается гауссовым распределением:

$$
I(q)=\frac{S}{2 \pi}\left(\frac{k}{q_{1}}\right)^{2} e^{-q^{2} /\left(2 q_{1}^{2}\right)},
$$


где $S$ - площадь образца и

$$
q_{1}^{2}=\frac{L}{l}\left(\frac{\alpha}{2 R}\right)^{2} \ln \frac{\alpha^{2} L}{2 l}
$$

Эти выражения описывают слегка модифицированное приближение “случайного блуждания" по углу [28].

Однако во второй области для $L \gg L_{0}$ выражение для интенсивности приобретает совершенно другой вид:

$$
I(q)=\frac{S q_{2} k^{2}}{2 \pi\left(q_{2}^{2}+q^{2}\right)^{3 / 2}},
$$

где

$$
q_{2}^{2}=\frac{L\left(1+\pi^{-1}\right)}{2 l R} .
$$

Теперь ширина распределения интенсивности $q_{2}$ пропорциональна толщине образца $L$. Таким образом, обнаружено сильное отклонение от поведения типа "случайного блуждания". Легко видеть, что хвост распределения интенсивности определяется однократным рассеянием и спадает как $q^{-3}$. В то же время полная интенсивность в области больших $q$ не зависит от толщины образца в противоположность однократному рассеянию. Очевидно, что такое расщепление толщины образца на две области и $q^{-3}$-поведение интенсивности для больших $q$ не является следствием сферической формы частиц и должно иметь место в общем случае разбавленных систем с характерным размером $R$.

Итак, при исследовании малоуглового рассеяния нейтронов на крупномасштабных неоднородностях большое значение имеет величина борновского параметра $\alpha$. Условия $\alpha \ll 1$ и $\alpha \gg 1$ соответствуют двум разным физическим ситуациям. В первом случае (область дифракции) нейтроны дифрагируют на неоднородностях, а во втором (область рефракции), проходя через них, испытывают преломление. Однократное рассеяние в области дифракции происходит на характерный угол $\Delta \theta_{\mathrm{D}} \sim \lambda / R$. Интенсивность рассеяния $\sim q^{-n}$ с показателем, близким к 4 , который зависит от природы неоднородности. Полное сечение рассеяния является малой величиной $\left(\sim \alpha^{2}\right)$, и длина свободного пробега нейтрона много больше, чем расстояние между рассеивателями, даже для плотных систем. Следовательно, в этом случае имеет место многократное рассеяние. Интенсивность многократного рассеяния нейтронов на неоднородностях описывается гауссовой функцией распределения по углам с характерным углом $\sim \sqrt{L}$, где $L-$ толщина образца. Однократное рассеяние в области рефракции происходит на характерный угол $\Delta \theta_{\mathrm{R}} \sim \lambda^{2} N \bar{b}$. Здесь полное сечение рассеяния не содержит малого параметра, и поэтому многократное рассеяние имеет место только в случае разбавленных систем. В зависимости от толщины образца существуют две области. В первой (для малых толщин образца) интенсивность многократного рассеяния также описывается гауссовой функцией, но характерный угол $\sim[(L / l) \ln (L / l)]^{1 / 2}$. Во второй области (для больших толщин образца) характерный угол $\sim(L / 2 l R)$. Отметим, что многократная рефракция в плотных системах, вероятно, может быть рассмотрена как результат малых отклонений классической частицы, движущейся через набор случайно ориентированных интерфейсов. 


\section{4. ЗАКЛЮЧЕНИЕ}

Одним из самых значительных достижений Уральского нейтронографического центра является разработка теоретических основ и экспериментальных методов магнитной нейтронографии веществ - как регулярных кристаллов, так и различных сплавов.

В области теории создан эффективный метод расшифровки магнитных структур с использованием теории симметрии кристалла. Магнитная структура с заданным волновым вектором представляется в виде разложения по неприводимым представлениям пространственной группы, при этом определению подлежит не ориентация атомных магнитных моментов кристалла, а коэффициенты смешивания базисных функций. Сами же базисные функции вычисляются для кристалла, пространственная группа для которого известна, так же как позиции атомов в примитивной ячейке. Поскольку число коэффициентов смешивания невелико (оно определяется размерностью неприводимого представления), предлагаемый метод тем эффективней, чем сложнее исходный кристалл.

При этом предполагается, что фазовый переход происходит по одному неприводимому представлению в соответствии с гипотезой Ландау в теории фазовых переходов второго рода. Сотрудниками Института физики металлов УрО РАН проведен симметрийный анализ большого числа исследованных магнитных структур и сделан вывод, что они описываются одним неприводимым представлением. В то же время выявлены некоторые исключения из этого правила, и в ряде случаев дано симметрийное объяснение этим фактам. Одним из таких симметрийных факторов является тот факт, что симметрия обменного гамильтониана выше симметрии кристалла, поэтому в случае малой анизотропии магнитная структура может характеризоваться не неприводимым представлением пространственной группы, а неприводимым представлением обменной группы (обменным мультиплетом).

Предложенный симметрийный подход был широко использован группой исследователей из Кракова (Польша), издавших обширные справочники, в которых указаны ответственные неприводимые представления для всех известных в литературе магнитных структур. Разработанный уральскими физиками симметрийный анализ магнитных структур широко применяется нейтронографистами во всем мире. Разработана общая теория малоуглового рассеяния в неоднородных веществах, в которых существуют мелкодисперсные включения в магнитную матрицу.

Отметим, что предлагаемый обзор отмечает лишь главные направления исследований по исследованию магнитного состояния вещества, ведущихся в Уральском нейтронографическом центре. Однако по этим направлениям получены фундаментальные результаты, которые объединяют и теоретические, и экспериментальные методы исследования с помощью рассеяния медленных нейтронов.

Благодарности. Работа выполнена по плану РАН (тема № 0120106436, шифр "Импульс"), при частичной поддержке проекта РФФИ (грант № 10-02-00155) и проекта 09-Т-2-1012 УрО РАН.

\section{Список литературы}

[1] С. В. Тябликов, Укр. матем. журн., 11 (1959), 287-294.

[2] Н. Н. Боголюбов, С. В. Тябликов, Докл. АН СССР, 126:1 (1959), 53-56. 
[3] В. Л. Бонч-Бруевич, С. В. Тябликов, Метод функиий Грина в статистической механике, Физматлит, М., 1961.

[4] Ю. А. Изюмов, Р. П. Озеров, Магнитная нейтронография, Наука, М., 1966.

[5] С. В. Тябликов, Методы квантовой теории магнетизма, Наука, М., 1965.

[6] С.В. Тябликов, Методы квантовой теории магнетизма, 2-е изд., испр. и дополн., Наука, М., 1975.

[7] В. Г. Вакс, А. И. Ларкин, С. А. Пикин, ЖЭТФ, 53:1 (1967), 281-299.

[8] В. Г. Вакс, А. И. Ларкин, С. А. Пикин, ЖЭТФ, 53:3 (1967), 1089-1106.

[9] А. А. Абрикосов, Л. П. Горьков, И. Е. Дзялошинский, Методы квантовой теории поля в статистической физике, Наука, М., 1962.

[10] Ю.А. Изюмов, Ф. А. Кассан-Оглы, Ю. Н. Скрябин, Полевые методы в теории ферромагнетизма, Наука, М., 1974.

[11] Ю.А. Изюмов, Ю.Н. Скрябин, Статистическая механика магнито-упорядоченных систем, Наука, М., 1987.

[12] Ю. А. Изюмов, М. И. Кацнельсон, Ю. Н. Скрябин, Магнетизм коллективизированных электронов, Наука, М., 1994.

[13] Ю.А. Изюмов, В.Е. Найш, Р.П. Озеров, Нейтронография магнетиков, Атомиздат, M., 1981.

[14] Ю. А. Изюмов, Н. А. Черноплеков, Нейтронная спектроскопия, Энергоатомиздат, М., 1983.

[15] Ю.А. Изюмов, Дифракиия нейтронов на длиннопериодических структурах, Энергоатомиздат, М., 1987.

[16] Ю.А. Изюмов, УФН, 131 (1980), 387-422.

[17] Ю. А. Изюмов, УФН, 144 (1984), 439-474.

[18] Ю. А. Изюмов, Ю. Н. Скрябин, "Нейтронография магнитных структур", 2010, Статья, посвященная 100-летию академика С. В. Вонсовского. Не опубликовано.

[19] О.В. Ковалев, Неприводимые представления пространственных групп, Изд-во АН УССР, Киев, 1961.

[20] Е. М. Лифшиц, ЖЭЭТФ, 11 (1941), 255-268.

[21] Ю.А. Изюмов, В.Е. Найш, С.Б. Петров, В.Н. Сыромятников, $Ф M M, 47$ (1979), 231-246; 455-463; 679-688

[22] Yu. A. Izyumov, V. E. Naish, V. N. Syromyatnikov, JMMM, 12:3 (1979), 249-261.

[23] Yu. A. Izyumov, V.E. Naish, JMMM, 12:3 (1979), 239-248.

[24] Л. Д. Ландау, Е. М. Лифшиц, Теоретическая физика, в 10 т., т. III: Квантовая механика (нерелятивистская теория), ред. Л. П. Питаевский, Наука, М., 1989.

[25] Y. N. Skryabin, Solid State Commun., 88:9 (1993), 747-748.

[26] R. J. Weiss, Phys. Rev., 83:2 (1951), 379-389.

[27] D. J. Hughes, M. T. Burgy, R. B. Heller, J. W. Wallace, Phys. Rev., 75:4 (1949), 565-569.

[28] С. В. Малеев, Б. П. Топерверг, ЖЭТФ, 78:1 (1980), 315-330.

[29] S. V. Maleyev, R. V. Pomortsev, Yu. N. Skryabin, Phys. Rev. B, 50:10 (1994), 7173-7176. 